\title{
Paroniceras, Frechiella y Oxyparoniceras (Ammonitida) en relación con las fluctuaciones térmicas y los ciclos transgresivo-regresivos detectados en el Toarciense de España
}

\author{
Gemma MARTÍNEZ
}

Departamento de Paleontología, Facultad de Ciencias Geológicas. Universidad Complutense de Madrid. Calle José Antonio Nováis, 12, 28040 Madrid, España; gemmamar@ucm.es

Martínez, G. 2017. Paroniceras, Frechiella y Oxyparoniceras (Ammonitida) en relación con las fluctuaciones térmicas y los ciclos transgresivo-regresivos detectados en el Toarciense de España. [Paroniceras, Frechiella, and Oxyparoniceras (Ammonitida) related with palaeoclimate fluctuations and regressive-transgressive cycles in the Toarcian of Spain]. Spanish Journal of Palaeontology, 32 (1), 109-114.

\section{RESUMEN}

El registro de las especies de Frechiella Prinz, 1904, Paroniceras Bonarelli, 1893 y Oxyparoniceras Guex, 1974 durante el Toarciense en el E y N de España, se ha relacionado con la evolución paleogeográfica y los ciclos deposicionales reconocidos en la Cordillera Ibérica. Sin embargo, es más evidente su relación con los intervalos de calentamiento relativo y picos térmicos, indicados por distintos autores en el Toarciense del norte y de la parte centro-oriental de la Subplaca Ibérica. Frechiella se ha registrado en la Zona Bifrons, Paroniceras en la Zona Variabilis y Oxyparoniceras en la parte superior de la Zona Thouarsense y parte inferior de la Zona Dispansum. La relación entre el registro de dichos géneros y las paleotemperaturas, parece indicar que sus especies se han desarrollado en medios cálidos y su presencia en las Cordilleras Ibérica y Cantábrica se debe a una migración desde otras áreas, sin que en todos los casos esto implique una colonización efectiva.

Palabras clave: Hildoceratidae, paleogeografía, paleoclimatología, Jurásico, E y $\mathrm{N}$ de España.

\begin{abstract}
The record of species of Frechiella Prinz 1904, Paroniceras Bonarelli 1893, and Oxyparoniceras Guex 1974, throughout the Toarcian in the east and north of Spain, has been linked with the palaeogeographic evolution and the depositional cycles recognized in the Iberian Chain. However, the relationship between this record and the relative warming intervals and thermal peaks, pointed out by several authors in the Toarcian of Northern and East-Central part of the Iberian Subplate, is more evident. Frechiella has been recorded in the Bifrons Zone, Paroniceras in the Variabilis Zone, and Oxyparoniceras in the upper part of the Thouarsense Zone and the lower part of the Dispansum Zone. The relationships between the record of these genera and the palaeotemperatures may indicate that their species developed in warm environments. Thus, their presence in the Iberian and Cantabrian chains could be due to a migration from other areas, but not to an effective colonization.
\end{abstract}

Keywords: Hildoceratidae, palaeogeography, palaeoclimatology, Jurassic, E and N Spain. 


\section{INTRODUCCIÓN}

Los géneros Frechiella, Paroniceras y Oxyparoniceras (= Jacobella Jeannet in Howarth, 2013) han sido objeto de diferentes trabajos y monografías llevados a cabo por Rulleau et al. (2003), Venturi \& Bilotta (2008), Goy \& Martínez (2009), Kovács (2010), Howarth (2013) y Martínez \& Comas Rengifo (2013) entre otros, sobre ammonoideos del Toarciense. Su clasificación taxonómica es controvertida y ha sufrido numerosos cambios. En trabajos recientes, se han incluido en la familia Paroniceratidae y más recientemente en Bouleiceratinae, familia Hildoceratidae (ver Howath, 2013), atendiendo principalmente a su línea de sutura simplificada, a pesar de su heterogeneidad morfológica.

El número de ejemplares identificados de los tres géneros en los materiales del Toarciense de la Península Ibérica es escaso en relación con el número total de los ammonoideos reconocidos y, en conjunto, constituyen menos del 1\% de los ammonoideos del Toarciense (Goy \& Martínez, 2009). Sin embargo, tienen una presencia mayor en otras áreas del Tethys (Rulleau et al., 2003; Goy \& Martínez, 2009; Kovács, 2010). Su distribución estratigráfica se ha obtenido a partir del estudio detallado de 18 secciones expandidas, representativas del NE España (Fig. 1).

\section{CONSIDERACIONES CRONOESTRATIGRÁFICAS, PALEOGEOGRÁFICAS Y PALEOCLIMÁTICAS}

El género Frechiella (especie tipo: Nautilus subcarinatus Young \& Bird, 1822) incluye morfologías globosas, con ombligos estrechos y línea de sutura sencilla (Figs 2 a-c). En España se registra en la Zona Bifrons (Subzona Sublevisoni y parte inferior de la Subzona Bifrons), coincidiendo con un episodio transgresivo que representa el momento de máxima profundización de todo el Jurásico Inferior en éste área (Gómez \& Goy, 2000, 2005; Aurell et al., 2003), y que corresponde a un intervalo de calentamiento según los estudios realizados por Gómez et al. (2008) (Fig. 3). Su posición estratigráfica es similar a la que presenta en otras cuencas de Portugal, Inglaterra, Francia y Hungría (Mouterde, 1967; Howarth, 1992; Elmi et al., 1997; Rulleau et al., 2003; Géczy \& Szente, 2006; Kovács, 2010).

En el género Paroniceras (especie tipo: Paroniceras sternale (v. Buch in d'Orbigny, 1842) se incluyen morfologías globosas con vuelta de espira de sección redondeada, con ombligo muy cerrado, ornamentación

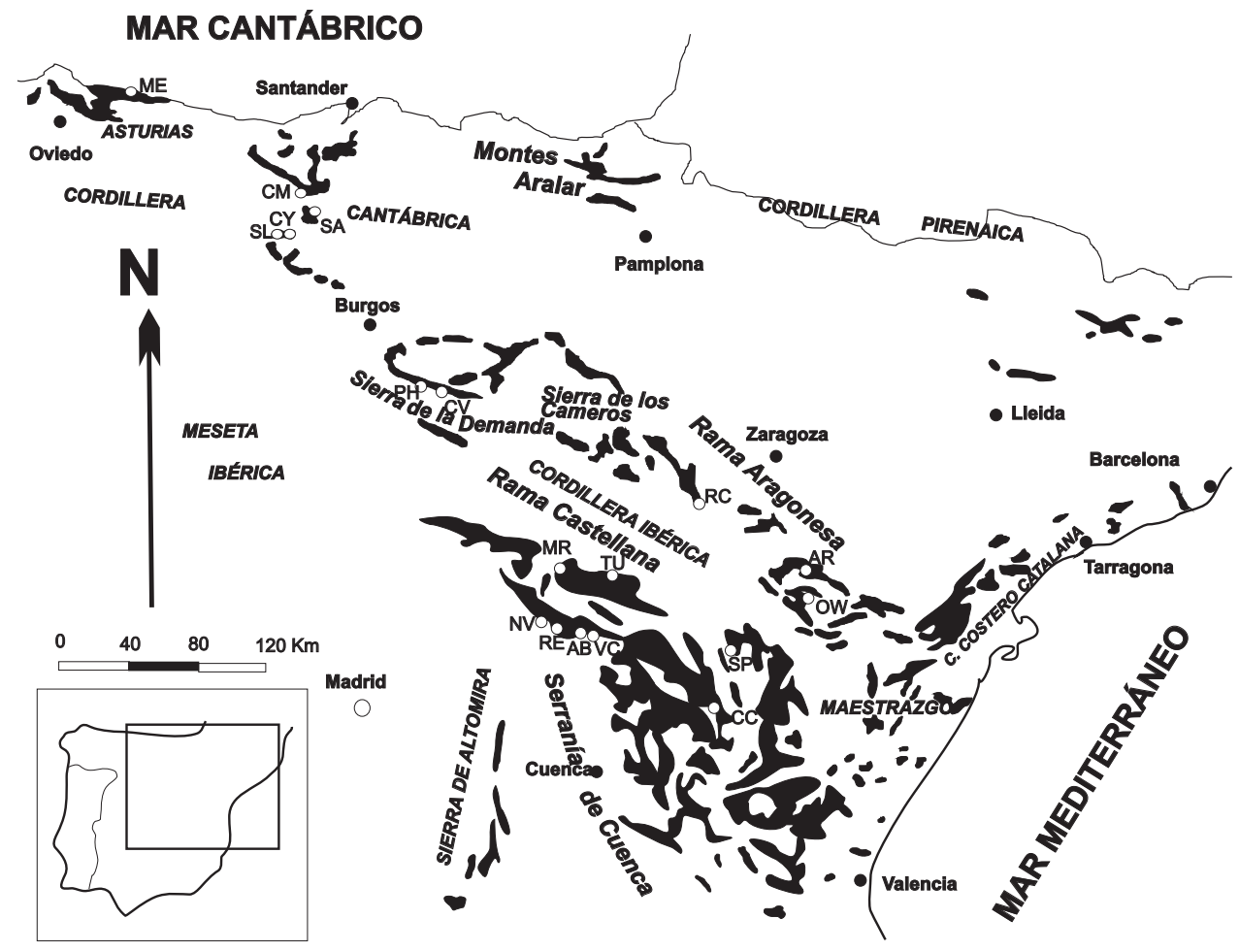

Figura 1. Afloramientos de los materiales del Jurásico Inferior en las cordilleras Ibérica y Cantábrica (en negro) y localización de las secciones estudiadas en Goy \& Martínez (2009). Abreviaturas: AR, Ariño; OW, Obón; SP, Sierra Palomera; CC, CoscojarEntrambasaguas; TU, Turmiel; MR, Maranchón; NV, Navalpotro; RE, Renales; AB, Ablanquejo; VC, Villar de Cobeta; RI, Ricla; CA, Castrovido; PH, Piedrahita de Muño; SL, Salinas de Pisuerga; CY, Cillamayor; SA, San Andrés; CM, Camino; ME, Santa Mera. 

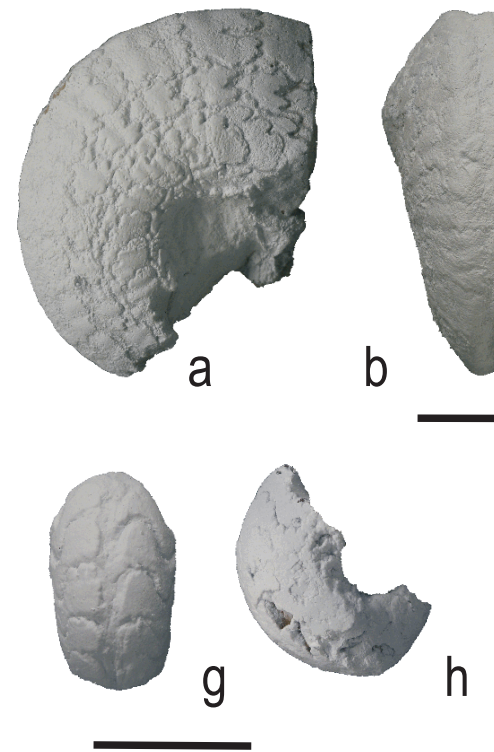

d
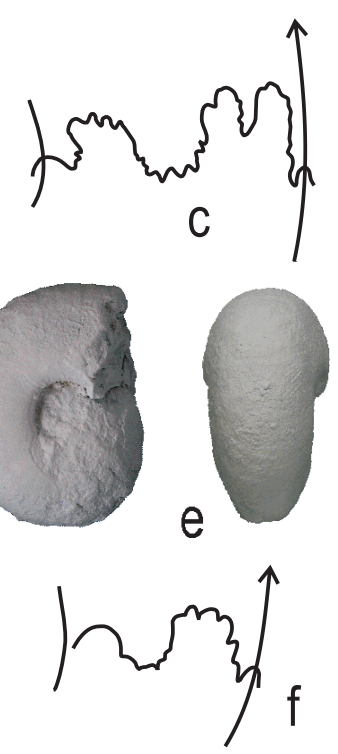

Figura 2. a-b) Frechiella subcarinata (Young \& Bird) AB6.3c/3, Subzona Sublevisoni, Ablanquejo, Guadalajara. c) Frechiella subcarinata (Young \& Bird) TU17.2/1, Subzona Sublevisoni, línea de sutura. $\mathrm{H}$ $=23,5 \mathrm{~mm}$. d-e) Paroniceras sternale (d'Orbigny) 2AR193/1, Subzona Variabilis, Ariño, Teruel. f) Paroniceras sternale (d'Orbigny) RE11.1/3, Zona Variabilis, Renales, Guadalajara, línea de sutura. $\mathrm{H}$ $=16,8 \mathrm{~mm}$. g-h) Oxyparoniceras $(O.) \mathrm{cf}$. telemachi (Renz), SL-L5.5/1 (x1,5), Subzona Fallaciosum, Salinas de Pisuerga, Palencia.

Frechiella subcarinata (Young \& Bird) F. helenae Renz

F. cf. octaviae Renz

F. kammerkarensis (Stolley)

F. venantii (Catullo)

Paroniceras sternale (d'Orbigny)

$P$. helveticum Renz

Paroniceras sp.

O. (Oxyparoniceras) cf. telemachi

0. (0.) buckmani (Bonarelli)

O. (0.) suevicum (Renz)

Oxyparoniceras sp.

O. (Neoparoniceras) cf. undulosum (Mon)

O. (N.) cf. morbiense (Renz)

O. (N.) cf. brocardi Rulleau, Bécaud, Neige

Frechiella Prinz

Paroniceras Bonarelli

Oxyparoniceras Renz

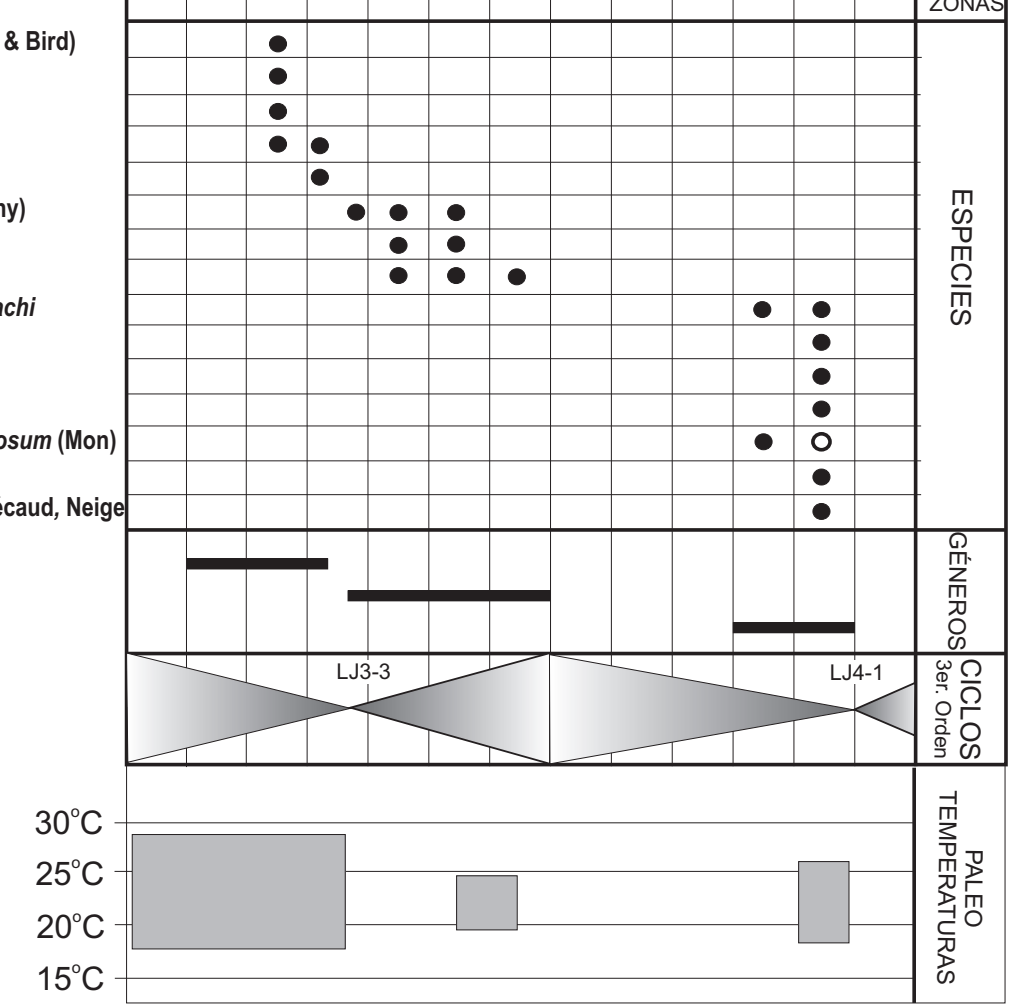

Figura 3. Distribución cronoestratigráfica de las especies de Frechiella, Paroniceras y Oxyparoniceras registradas en el E y N de España (modificado de Goy \& Martínez, 2009). Círculos negros: presencia confirmada; círculo blanco: presencia probable. Se incluye el registro obtenido de Paroniceratinae en relación con los ciclos transgresivo-regresivo, caracterizados por Gómez \& Goy $(2004,2005)$ en el E de España y con los intervalos y picos térmicos detectados por Gómez et al. (2008). Abreviaturas de las subzonas: EL, Elegantulum; FA, Falciferum; SU, Sublevisoni; BI, Bifrons; SM, Semipolitum; VA, Variabilis; IL, Illustris; VI, Vitiosa; BN, Bingmanni; TH, Thouarsense; FL, Fallaciosum; IN, Insigne; GR, Gruneri. (Modificado de Goy \& Martínez, 2009). 
muy tenue y línea de sutura simple, de estilo ceratítico (Figs 2 d-f). En España se ha reconocido en materiales del intervalo comprendido entre la Zona Bifrons (Subzona Semipolitum) y la Zona Variabilis (Subzona Vitiosa). Su registro en la Cuenca Ibérica coincide con el inicio y desarrollo progresivo de un episodio de somerización en el borde oriental de la Subplaca Ibérica (Gómez \& Goy, 2005) y con un pico térmico en este área (Gómez et al., 2008) (Fig. 3). Este género es muy frecuente en el Tethys, donde han sido descritas numerosas especies y variedades (Teruzzi, 1981; Cresta et al., 1995; Kovács, 2010) y fuera de él se ha reconocido en cuencas centroeuropeas (ver Rulleau et al., 2003) y de América del Norte (Tipper et al., 1991; Jakobs, 1995), dentro de una banda latitudinal bastante reducida (Fig. 4).

En el género Oxyparoniceras (especie tipo: Paroniceras telemachi (Renz, 1912) se incluyen dos subgéneros: $O$. (Neoparoniceras) y O. (Oxyparoniceras). Se trata de conchas muy involutas, más o menos comprimidas, con la sección ojival, flancos fuertemente convergentes, región ventral carenada o no, con ornamentación compuesta de simples estrías radiadas y línea de sutura simple, próxima a la de Paroniceras (Figs 2 g, h). En España tiene una escasa presencia, encontrándose de forma esporádica en las zonas Thouarsense y Dispansum. Su registro coincide con un episodio transgresivo (Gómez \& Goy, 2005; Quesada et al., 2005) que representa una nueva etapa de profundización relativa, con su máxima expresión en la parte inferior de la Subzona Insigne, que en las localidades en que se registra este género supone un ambiente de plataforma donde se alcanzaron batimetrías relativamente elevadas. También coincide con un nuevo incremento de la temperatura (Gómez et al., 2008) (Fig. 3). Fuera de España ha sido citada en el ámbito del Tethys, en diversas localidades de la Provincia NO Europea y en el norte de África (Rulleau et al., 2003; Goy \& Martínez, 2009) (Fig. 4).

\section{DISCUSIÓN Y CONCLUSIONES}

Las características morfológicas de los géneros, especialmente en lo que se refiere a la línea de sutura, parecen corresponder a un tipo de vida nectónica en aguas poco profundas. Su presencia en las cuencas estudiadas se puede relacionar con la evolución paleogeográfica de las mismas y con los ciclos deposicionales reconocidos en la Cordillera Ibérica. Frechiella y Oxyparoniceras se han encontrado en sedimentos de la Zona Bifrons y de las zonas Thouarsense e Insigne respectivamente, coincidiendo
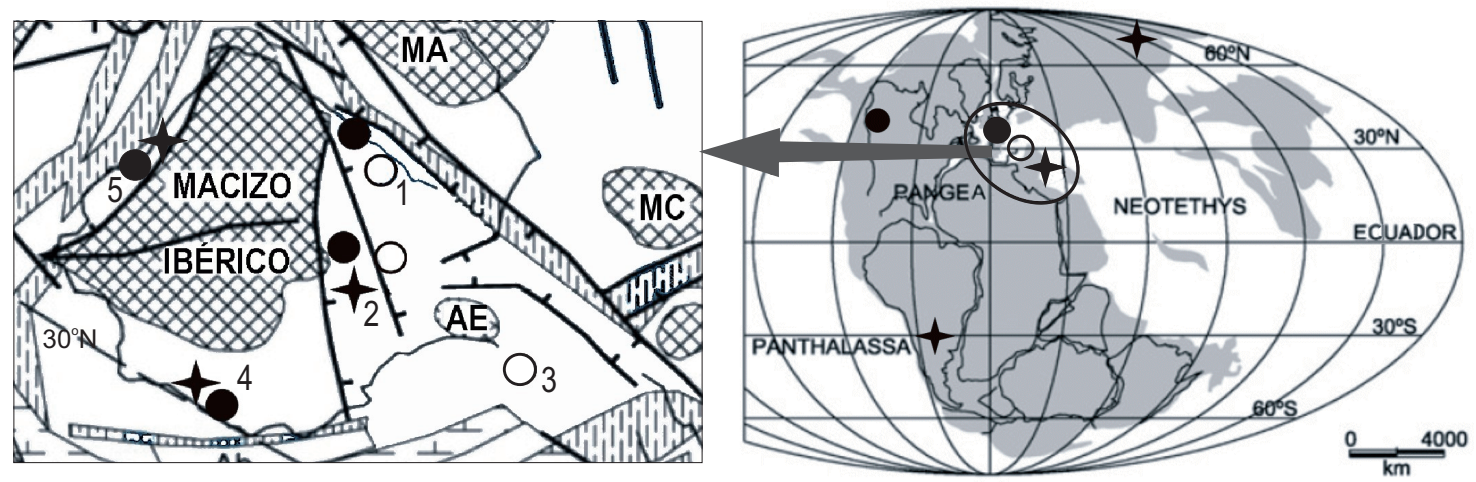

Alto cratónico
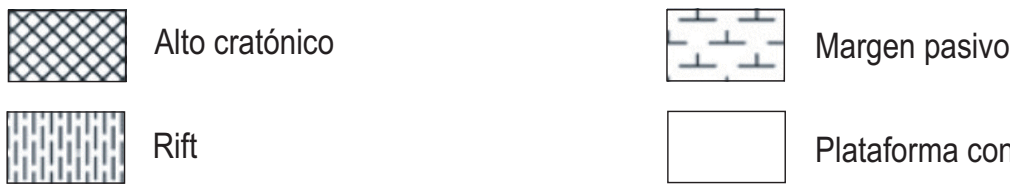

Rift

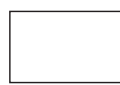

Plataforma continental y cuenca oceánica

Paroniceras
Oxyparoniceras

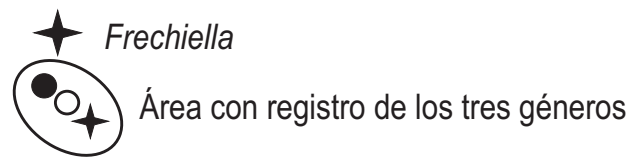

Figura 4. Mapa paleogeográfico (tomado de Goy \& Martínez, 2009; modificado de Gómez et al., 2008) con la distribución de los géneros estudiados en este trabajo. MA, Macizo Armoricano; MC, Macizo Central; AE, Alto del Ebro. 1, Cordillera Cantábrica; 2, Cordillera Ibérica; 3, Isla de Mallorca; 4, Cordillera Bética; 5, Cuenca Lusitánica. 
con máximos transgresivos, mientras que Paroniceras procede de la Zona Variabilis correspondiente al inicio de un evento regresivo. Por otra parte, las variaciones de las paleotemperaturas detectadas en el $\mathrm{E}$ y $\mathrm{N}$ de España indican un aumento relativo de la temperatura en las subzonas Sublevisoni y Bifrons, y de manera menos acentuada en las subzonas Illustris y Vitiosa, donde se registran las especies de Frechiella y Paroniceras, y en la Subzona Insigne, donde se encuentran todas las especies del género Oxyparoniceras. Por todo ello, parece que la presencia/ ausencia de Frechiella, Paroniceras y Oxyparoniceras, así como la variación de sus caracteres morfológicos, estarían más relacionadas con los incrementos térmicos detectados en las diferentes cuencas, apropiados para el desarrollo de estos géneros, que con los cambios y fluctuaciones en las condiciones paleogeográficas y paleobatimétricas. Por otra parte, su registro escaso y esporádico hace suponer que no hubo una colonización efectiva. Probablemente se trata de inmigrantes ocasionales procedentes de otras áreas del Tethys occidental situadas en paleolatitudes similares a las de la Península Ibérica durante el Toarciense, dentro del ámbito mediterráneo central y oriental, donde su registro es notablemente más abundante (Rulleau et al., 2003, p. 324).

\section{AGRADECIMIENTOS}

Agradecer y reconocer la gran labor científica del Profesor Pascual Rivas Carrera, cuyos trabajos son un claro referente en el mundo de la Paleontología. Este trabajo se ha visto beneficiado por los comentarios y sugerencias de los profesores Antonio Goy Goy, $\mathrm{M}^{\mathrm{a}}$ José Comas Rengifo y Fernando García Joral, y de los revisores del mismo, Juan Carlos Braga Alarcón y José Sandoval Gabarrón. Este trabajo es una contribución a los Proyectos CGL201123947, CGL2011-25894, CGL2015-66604-R y al Grupo de Investigación UCM Procesos Bióticos Mesozoicos.

\section{REFERENCIAS}

Aurell, M., Robles, S., Bádenas, B., Rosales, I., Quesada, S., Meléndez, G. \& García-Ramos, J.C. 2003. Transgressiveregressive cycles and Jurassic palaeogeography of northeast Iberia. Sedimentary Geology, 162, 239-271; doi: 10.1016/S0037-0738(03)00154-4.

Bonarelli, G. 1893. Osservazioni sul Toarciano e l'Aaleniani dell'Appenino centrale. Bolletino della Società Geologica italiana, 12, 195-254.

Cresta, S., Basilici, G., Bussoletti, A., Comas, M.J., Elmi, S., Fernández-Lopez, S., Ferreti, A., Goy, A., Henriques, M.H., Kalin, O., Jiménez, A., Martínez, G., Sandoval, J., Ureta, S. \& Venturi, F. 1995. Relazione sul workshop
«Paleontologia e biostratigrafia dei sedimenti ammonitiferi toarciano-bajociani in successioni di alto morfostrutturale dell'Apennino umbro-marchigiano: 1'area di Monte Nerone. Correlazioni con altreregioni della Tetide occidentale». Bolletino Servizio Geologico Italia, 112, $1-12$.

Elmi, S., Rulleau, L., Gabilly, J. \& Mouterde, R. 1997. Toarcien. In: Groupe Français d'Études du Jurassique: Biostratigraphie du Jurassique Ouest-Européen et Méditerranéen. Zonation Parallèle et Distribution des Invertébrés et Microfossiles Cariou. (ed. Hantzpergue, P.). Bulletin du Centre de Recherches Elf Exploration Production, 17, 25-36.

Géczy, B. \& Szente, I. 2006. Middle Toarcian Ammonitina from the Gerecse Mts, Hungary. Acta Geologica Hungarica, 49, 223-252.

Gómez, J.J. \& Goy, A. 2000. Definition and organization of limestone-marl cycles in the Toarcian of northern and eastcentral part of the Iberian Subplate (Spain). GeoResearch Forum, 6, 301-310.

Gómez, J.J. \& Goy, A. 2004. Jurásico Inferior de las cordilleras Ibérica y Costero-Catalana. In: Geología de España (ed. Vera, J.A.). SGE-IGME, Madrid, pp. 495-500.

Gómez, J.J. \& Goy, A. 2005. Late Triassic and Early Jurassic palaeogeographic evolution and depositional cycles of the Western Tethys Iberian platform system (Eastern Spain). Palaeogeography, Palaeoclimatology, Palaeoecology, 222, 77-94; doi: 10.1016/j.palaeo.2005.03.010.

Gómez, J.J., Goy, A. \& Canales, M.L. 2008. Seawater temperature and carbon isotope variations in belemnites linked to mass extinction during the Toarcian (Early Jurassic) in Central and Northern Spain. Comparison with other European sections. Palaeogeography, Palaeoclimatology, Palaeoecology, 258, 28-58; doi: 10.1016/j.palaeo.2007.11.005.

Goy, A. \& Martínez, G. 2009. Paroniceratinae (Ammonoidea, Hildoceratidae) del Toarciense en las Cordilleras Ibérica y Cantábrica (España). Geobios, 42, 603-622; doi: 10.1016/j.geobios.2009.03.004.

Guex, J. 1974. Les Bouleiceratinae Arkell, sous-famille mono ou polyphylétique? Eclogae geologicae Helvetiae, 67, 97-129.

Howarth, M.K., 1992. The ammonite family Hildoceratidae in the Lower Jurassic of Britain. Monograph of the Paleontographical Society part 1 (1991), 1-106; part 2 (1992), 107-200.

Howarth, M.K. 2013. Part L, Revised, Volume 3B, Chapter 4: Psiloceratoidea, Eodoceratoidea, Hildoceratoidea. KU University Institute, University of Kansas, Lawrence, Kansas, USA. Treatise Online, 57, 1-139.

Jakobs, G.K. 1995. New occurrence of Leukadiella and Paroniceras from the Toarcian of the Canadian Cordillera. Journal of Paleontology, 69, 89-98.

Kovács, Z. 2010. Paroniceratidae (Ammonitina) of the Toarcian from the Gerecse Mts (NE Transdanubian Range, Hungary). Hungarian Geological Society, 140, 119-134.

Martínez, G. \& Comas Rengifo, M.J. 2013. Paroniceratinae (Ammonoidea, Hildoceratidae) del Toarciense en las 
Cordilleras Ibérica y Cantábrica (España). XXIX Jornadas de Paleontología. Sociedad Española de Paleontología, Libro de Resúmenes, 91-92.

Mouterde, R. 1967. Le Lias de Portugal. Vue d'ensemble et division en Zones. Comunicaçoes dos Serviços Géologicos de Portugal, 52, 209-226.

Orbigny, A. 1842. Paléontologie Française. Terrains Jurassiques, 1 Céphalopodes. Masson, Paris.

Prinz, G. 1904. Die fauna die alteren jurabildungenim nordöstlichen Bakony. Mittei Jahrbuch königlich Ungaria geológica Anstalt, 15, 1-142.

Quesada, S., Robles, S. \& Rosales, I. 2005. Depositional architecture and transgressive-regressive cycles within Liassic backstepping carbonate ramps in the BasqueCantabrian Basin, northern Spain. Journal of Geological Society London, 162, 531-548; doi: 10.1144/0016764903-041.

Renz, C. 1912. Neuere Fortschritte in ther Geologie und Paläontologie Griechlands. Zeitschrift der Deutschen Geologischen Gesellschaft, 64, 530-630.

Rulleau, L., Bécaud, M. \& Neige, P. 2003. Les ammonites traditionnellement regroupées dans la sous-famille des
Bouleiceratinae (Hildoceratidae, Toarcien): aspects phylogénétiques, biogéographiques et systématiques. Geobios, 36, 317-348; doi: 10.1016/S00166995(03)00034-2.

Teruzzi, G. 1981. Nota sulle ammoniti dei generi Frechiella, Paroniceras, Oxyparoniceras del Toarciano mediterraneo. Atti della Società Italiana di Scienze naturali e del Museo Civico di Storia naturali di Milano, 122, 21-31.

Tipper, H.W., Smith, P.L., Cameron, B.E.B., Carter, E.S., Jacobs, G.K. \& Johns, M.J. 1991. Biostratigraphy of the Lower Jurassic formations of the Queen Charlotte Islands, British Columbia. Geological Survey of Canada, 90, 203-235.

Venturi, F. \& Bilotta, M. 2008. New data and hypotheses on early Jurassic ammonite phylogeny. Revue de Paléobiologie, 27, 859-901.

Young, G. \& Bird, J. 1822. A Geological Survey of the Yorkshire Coast: Describing the Strata and Fossils Occurring between the Humber and the Tees, from the German Ocean to the Plain of York. 2nd edition, Whitby, Cambridge. 